\title{
Um Novo Esquema de ARQ Híbrido Para Códigos Turbo Com Combinação Por Diversidade e Transmissão Parcial
}

\author{
André Gustavo Degraf Uchôa, Richard Demo Souza e Marcelo Eduardo Pellenz
}

\begin{abstract}
Resumo-Um novo esquema de requisição de repetição automática híbrida (HARQ) utilizando códigos turbo é proposto. O método utiliza conjuntamente combinação por diversidade, retransmissão parcial e controle de potência. Simulações e análises baseadas em evolução da densidade mostram que o novo método supera o esquema clássico baseado em combinação por ganho igual e também esquemas baseados em combinação de informação suave para uma grande gama de valores de relação sinal-ruído.
\end{abstract}

Palavras-Chave-Códigos Turbo, Códigos Corretores de Erro, Requisição de Repetição Automática (ARQ), Requisição de Repetição Automática Híbrida (HARQ)

Abstract-A novel Hybrid Automatic Repeat reQuest (HARQ) scheme using turbo codes is proposed. The method jointly utilizes diversity combining, partial retransmission, and power control. Computer simulations and density evolution analysis show that the new method outperforms equal gain diversity combining and soft information combining techniques for a wide signal to noise range.

Keywords-Turbo codes, Error Correction Codes, Automatic Repeat Request (ARQ), Hybrid Automatic Repeat Request (HARQ)

\section{INTRODUÇÃO}

Muitos padrões modernos de comunicação suportam esquemas de requisição de repetição automática híbrida (HARQ, do inglês Hybrid Automatic Repeat reQuest) [1] usando códigos turbo [2] para uma transmissão praticamente livre de erros. Entre estes padrões podemos citar o HSDPA (do inglês High Speed Downlink Packet Access) [3], o WiMAX Móvel (IEEE 802.16e) [4], e o 3GPP LTE (do inglês 3GPP Long Term Evolution) [5].

Em esquemas HARQ que usam códigos turbo existem muitas maneiras de definir as retransmissões e a forma como estas serão combinadas no receptor, tais como em [6]-[9]. Classicamente, as maneiras de definir as retransmissões e combinações dos pacotes recebidos podem ser agrupadas como esquemas de redundância incremental (IR) ou combinação por diversidade (DC) [1]. Estes últimos também são conhecidos como esquemas de combinação de Chase [10].

Nos métodos IR paridades adicionais são enviadas a cada retransmissão, reduzindo a taxa do código vista no receptor e

André Gustavo Degraf Uchôa e Richard Demo Souza estão vinculados ao CPGEI, UTFPR. Av. Sete de Setembro, 3165, Curitiba, PR, 80230-901, Brasil, Emails: agdu@ieee.org, richard@utfpr.edu.br

Marcelo Eduardo Pellenz está vinculado ao PPGIA, PUC-PR. Rua Imaculada Conceicão, 1155, Curitiba, PR, 80215-901, Brasil, E-mail: marcelo@ppgia.pucpr.br aumentando a probabilidade de sucesso na decodificação. Provavelmente o melhor esquema IR conhecido usando códigos turbo é o introduzido em [6], o qual é baseado no conceito de códigos puncionados de taxas compatíveis [11]. Em métodos DC, as retransmissões são baseadas nos mesmos bits codificados. O receptor combina as múltiplas cópias recebidas para produzir uma única palavra código e como resultado o decodificador opera sobre um código de taxa fixa. Além do mais, combinações de IR e DC podem ser projetadas, tal como o método em [9], o qual funciona como IR nas duas primeiras transmissões e como DC nas demais.

Muitas comparações entre esquemas IR e DC podem ser encontradas na literatura, tais como [12]-[17]. É de se supor que em geral métodos IR deveriam superar os DC, uma vez que os DC são apenas uma forma de código de repetição. Entretanto, tais comparações demonstram que sob várias condições práticas métodos DC operam consideravelmente próximos aos IR, ou até melhor. Em [12], [13] vários cenários considerando HSDPA são investigados, e a conclusão é de que em sistemas com enlaces adaptativos IR é apenas marginalmente melhor que DC. Em [14] os autores investigaram cenários considerando LTE com OFDMA, onde demonstraram que, para um projeto apropriado levando-se em conta as subportadoras e as técnicas de reagrupamento da constelação, a diferença entre IR e DC é reduzida significativamente. Em [15] é mostrado que no caso de um canal com desvanecimento quase-estático DC pode ser uma solução melhor do que IR. Por fim, recentemente em [16], [17] os autores investigam o desempenho de esquemas HARQ em cenários com múltiplas antenas transmissoras e receptoras, chegando também à conclusão que em alguns casos DC é até mesmo melhor do que IR.

Além disso, vale lembrar que num esquema IR típico o decodificador sempre opera na menor taxa possível (os bits puncionados no codificador são substituídos por apagamentos no decodificador). Como resultado, o decodificador IR requer um buffer maior e é mais complexo em termos de decodificação, uma vez que o esforço computacional de um decodificador de Viterbi ou BCJR é inversamente proporcional à taxa do código [18]. Finalmente, um buffer muito grande e uma taxa muito baixa resultará em um circuito grande que, de modo geral, corresponde a um maior consumo de energia; o que pode ser indesejável, especialmente em dispositivos de comunicação portáteis.

Neste artigo, consideramos uma restrição de projeto que é manter o receptor HARQ tão simples quanto possível mas com 
um bom desempenho. Baseado nos resultados em [12]-[17], esta restrição pode ser traduzida para um decodificador operando a uma taxa fixa e com um buffer de mesmo tamanho do pacote inicialmente transmitido. Assim sendo, nosso objetivo é projetar um método DC que supere um receptor DC típico, o qual por sua vez é baseado em combinação por ganho igual (EGC, do inglês Equal Gain Combining) [1]. Além do método EGC clássico, também incluiremos em nossas comparações esquemas baseados na troca de informação suave (LLR, do inglês Log Likelihood Ratio) entre retransmissões, onde o mesmo codificador e decodificador são utilizados para cada retransmissão, como os métodos em [7] e [8].

O esquema proposto em [7] foi o primeiro a lidar com a combinação de informação suave para HARQ usando códigos turbo, e ainda é o mais reconhecido até o presente. A decisão suave da última transmissão é usada como informação a priori para iniciar a decodificação em uma retransmissão. Por sua vez, no método apresentado em [8] os autores acumularam apenas as saídas suaves prévias do canal para calcular os valores a priori para uma retransmissão.

Introduzimos aqui uma estratégia nova de HARQ DC baseada em retransmissão parcial e controle de potência. Simulações computacionais mostram que o método proposto supera o EGC clássico e esquemas de combinação suave [7], [8]. Além do mais, conduzimos uma análise baseada em evolução da densidade (do inglês density evolution) [19] de modo a identificar as razões pelos quais o esquema proposto possui um bom desempenho.

O restante deste artigo está organizado da seguinte maneira. $\mathrm{Na}$ Seção II definimos o modelo do sistema, enquanto na Seção III descrevemos o esquema HARQ proposto. Resultados numéricos baseados em simulações computacionais são apresentados na Seção IV. Na Seção V investigamos o desempenho do método proposto baseado nas análises de evolução da densidade, enquanto que na Seção VI concluímos o artigo.

\section{Modelo do Sistema}

Considere um sistema de comunicação com canais direto e de retorno, como ilustrado na Figura 1, onde o canal direto é modelado como um canal AWGN com uma densidade espectral de potência de ruído $N_{0}$, e o canal de retorno é considerado sem ruído. Nosso objetivo é transmitir um bloco de $L$ bits equiprováveis e independentes, $\mathbf{u}=\left[u_{0} u_{1} \cdots u_{L-1}\right]$, usando modulação BPSK. Os bits são codificados por um codificador turbo sistemático paralelo típico [2] antes da transmissão, objetivando uma saída sistemática $\mathbf{u}$ e duas saídas de paridade $\mathbf{v}^{\mathbf{1}}=\left[v_{0}^{1} v_{1}^{1} \cdots v_{L-1}^{1}\right] \mathrm{e} \mathbf{v}^{\mathbf{2}}=\left[v_{0}^{2} v_{1}^{2} \cdots v_{L-1}^{2}\right]$. Assim, a taxa do código base é $R=1 / 3$. Se antes da transmissão as duas saídas de bits de paridade forem alternadamente puncionadas, então uma taxa de código de $R=1 / 2$ é obtida. Outras taxas de código podem ser alcançadas através de diferentes padrões de puncionamentos. A saída do receptor ê é uma estimativa dos bits de informação transmitidos originalmente u. Um máximo de cinco decodificações por transmissão são consideradas, e é assumido um critério de parada ideal para o término antecipado das iterações em caso de decodificação com sucesso.

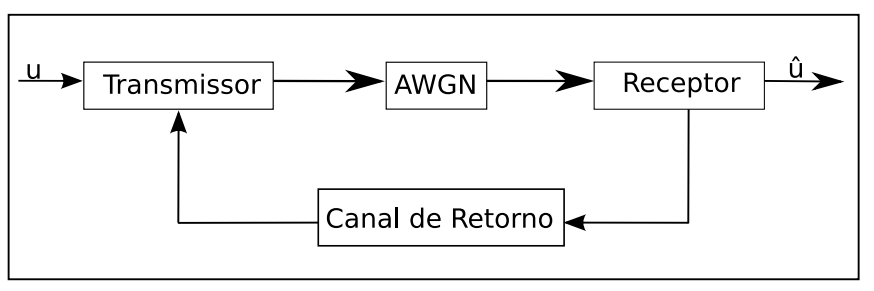

Fig. 1. Diagrama de blocos do sistema de comunicação com canais direto e de retorno.

Sem perda de generalidade, os esquemas ARQ que simulamos usam um protocolo simples stop-and-wait. Assumimos que os erros podem ser perfeitamente detectados pelo receptor. Quando um erro é detectado no receptor, uma requisição de retransmissão (NACK) é enviada para o transmissor. Quando um pacote é recebido com sucesso, um sinal de confirmação (ACK) é enviado para o transmissor, o qual segue para o próximo pacote. Um pacote é transmitido no máximo cinco vezes antes de ser considerado perdido.

Baseado em [1], consideramos a vazão como sendo $T=$ $E\left[\frac{k}{n^{\prime}}\right]$, onde $E[\cdot]$ denota o valor esperado e $n^{\prime}$ é o número total de bits codificados enviados (na transmissão original e em todas as retransmissões) para que um dado pacote seja aceito (corretamente decodificado) pelo receptor. Desta forma, para o caso de nenhum erro depois da decodificação da primeira transmissão, a vazão será igual à taxa do código $R=k / n$. Além do mais, uma vez que limitamos o número de retransmissões, e no caso do pacote não ser corretamente recebido até a última transmissão, a vazão instantânea é considerado zero, desta forma decrescendo a média da vazão.

\section{MÉtodo Proposto}

Nesta seção apresentamos a descrição do método proposto para duas taxas de código diferentes, $R=1 / 2$ e $R=1 / 3$.

\section{A. Taxa $R=1 / 2$}

Considere a seguinte estratégia de ARQ híbrido. Na primeira transmissão o sistema opera como usual, transmitindo um total de $2 \times L$ símbolos (correspondendo a $L$ bits sistemáticos e $L$ bits de paridade). Se uma segunda transmissão é necessária, então apenas os símbolos sistemáticos u são transmitidos. Assim, apenas $L$ símbolos são enviados na segunda transmissão. De forma a utilizar a mesma energia por transmissão, os símbolos são enviados com duas vezes a energia da que eles foram enviados na primeira transmissão, significando então que a magnitude dos símbolos são multiplicadas por $\sqrt{2}$. O receptor aplica combinação por diversidade (sempre por ganho igual) entre os símbolos sistemáticos recebidos previamente e os recém recebidos. Se uma terceira retransmissão se fizer necessária, então as paridades puncionadas $\mathbf{v}^{\mathbf{1}}$ e $\mathbf{v}^{\mathbf{2}}$ são enviadas (as mesmas paridades enviadas na primeira transmissão, tal que a taxa do código não é modificada). Novamente, apenas $L$ símbolos foram enviados através do canal. Com isso, de forma a manter a mesma energia usada por transmissão, nós dobramos a 
energia dos símbolos a serem enviados nesta transmissão. O receptor aplica combinação por diversidade com aquelas paridades recebidas na primeira transmissão. No caso de mais retransmissões se fazerem necessárias, o sistema alternará entre a transmissão de apenas símbolos sistemáticos e apenas paridades puncionadas, sempre aplicando o controle de potência e combinação por diversidade.

\section{B. Taxa $R=1 / 3$}

O método proposto pode ser estendido para outras taxas. Para o caso de taxa $R=1 / 3$ uma das opções é enviar apenas os bits sistemáticos $\mathbf{u}$ na primeira retransmissão, na segunda retransmissão apenas a primeira paridade $\mathbf{v}^{\mathbf{1}}$, e na terceira retransmissão enviar a segunda paridade $\mathbf{v}^{2}$. Se mais retransmissões são necessárias, o sistema repete a estratégia da primeira retransmissão à terceira. Uma vez que apenas um terço dos símbolos são enviados a cada retransmissão ( $L$ símbolos, ao invés de $3 \times L$ ), uma forma de usar a mesma energia por transmissão é incrementar a magnitude dos símbolos retransmitidos por um fator de $\sqrt{3}$.

Note que o método proposto, para ambas as taxas de código acima descritas, não pode ser classificado como um esquema HARQ IR Parcial. Em tais técnicas cada retransmissão é auto decodificável, e geralmente é uma sequência puncionada complementar do código mãe [20]. No método proposto, cada retransmissão não é auto decodificável e nem uma sequência puncionada complementar. Simplesmente retransmitimos partes do mesmo pacote que fora enviado na primeira transmissão, com uma alocação apropriada de energia, de forma que o decodificador vê um código de taxa fixa. Isto não acontece com esquemas HARQ IR Parciais, os quais são uma sub-classe de métodos IR.

\section{RESUltados NumÉRicos}

Nesta seção investigamos o desempenho do esquema proposto na Seção III em termos da vazão e da complexidade de decodificação, como função de $E_{b} / N_{0}$, onde $E_{b}$ é a energia por bit de informação. Nas seguintes simulações consideramos um $E_{b} / N_{0}$ fixo para todas as retransmissões, que é igual ao valor de $E_{b} / N_{0}$ na primeira transmissão. Além disso, utilizamos o código turbo com geradores $(17,15)$, em octal, um bloco de $L=256$ bits de informação, e a transmissão de 10000 blocos para cada valor de $E_{b} / N_{0}$.

A Figura 2 apresenta a vazão para o esquema proposto, para HARQ usando o mesmo código turbo e $\mathrm{EGC}^{1}$, para o método de combinação suave de [7], e o método de combinação suave de [8]. Na figura também é apresentada a vazão para um esquema HARQ regular sem combinação, onde os pacotes recebidos previamente são simplesmente descartados. Na figura pode-se notar que o método proposto apresenta uma vantagem clara sobre o método HARQ com EGC clássico para uma vasta gama de $E_{b} / N_{0}$, sendo quase coincidente para os casos

\footnotetext{
${ }^{1}$ Note que no caso de HARQ com EGC em cada retransmissão a palavra codificada completa é transmitida, ao contrário do método proposto onde apenas parte da palavra codificada é enviada em cada retransmissão. Esta operação baseada em transmissão parcial é que permite o controle de potência utilizado no método proposto.
}

de valores de $E_{b} / N_{0}$ muito baixos e muito altos. Para alguns valores de $E_{b} / N_{0}$ o ganho do esquema proposto chega a $2 \mathrm{~dB}$. Além disto, o método proposto e o HARQ com EGC superam os esquemas de combinação suave de [7] e [8], enquanto todos os métodos possuem um desempenho consideravelmente melhor que o HARQ regular sem combinação.

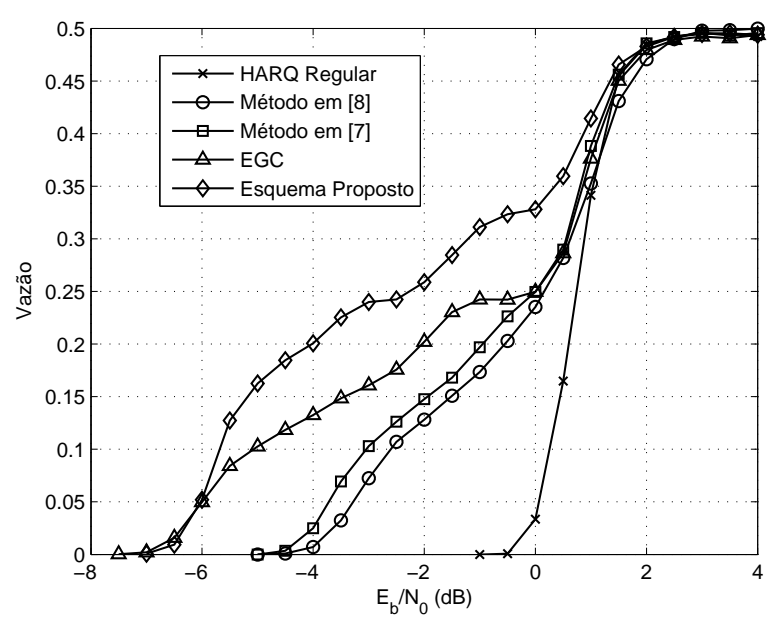

Fig. 2. Vazão versus $E_{b} / N_{0}$ para o esquema proposto, HARQ com EGC, os métodos de [7], [8], e HARQ regular sem combinação. A taxa do código é $R=1 / 2$.

A Figura 3 compara a média do número total de iterações requeridas para o esquema proposto e os outros métodos. O número total de iterações de decodificação é a soma do número de iterações utilizadas em cada retransmissão até que um pacote seja corretamente decodificado. Esta é uma boa medida para avaliar a complexidade computacional de diferentes esquemas, uma vez que todos eles usam o mesmo tipo de decodificador. Na figura nós podemos ver que o número médio de iterações de decodificação para o esquema proposto é muito próximo ao do HARQ com EGC clássico, e consideravelmente menor que para todos os outros métodos para valores baixos de $E_{b} / N_{0}$. Assim, o esquema proposto alcança um desempenho melhor que o HARQ com EGC com basicamente a mesma complexidade computacional.

Como pode ser visto na Figura 4 , que considera $R=1 / 3$, mais uma vez o esquema proposto é consideravelmente melhor em termos de vazão do que o HARQ com EGC clássico, e os métodos de combinação de informação suave de [7], [8], para uma grande gama de valores de $E_{b} / N_{0}$. Os ganhos sobre o HARQ com EGC são maiores do que no caso de taxa $R=$ $1 / 2$, sendo tão grandes quanto $3 \mathrm{~dB}$ para alguns valores da razão $E_{b} / N_{0}$.

A Figura 5 apresenta o número médio do total de iterações de decodificação para o caso de taxa $R=1 / 3$ para todos os métodos em análise. Na figura podemos ver que mais uma vez a complexidade de decodificação para o esquema proposto é muito próxima da do HARQ com EGC, como visto para o caso da taxa $R=1 / 2$. Como neste caso, ambos os métodos são muito menos complexos que os esquemas em [7], [8] e HARQ regular sem combinação. A melhoria de desempenho do método proposto não acarreta em um aumento considerável 


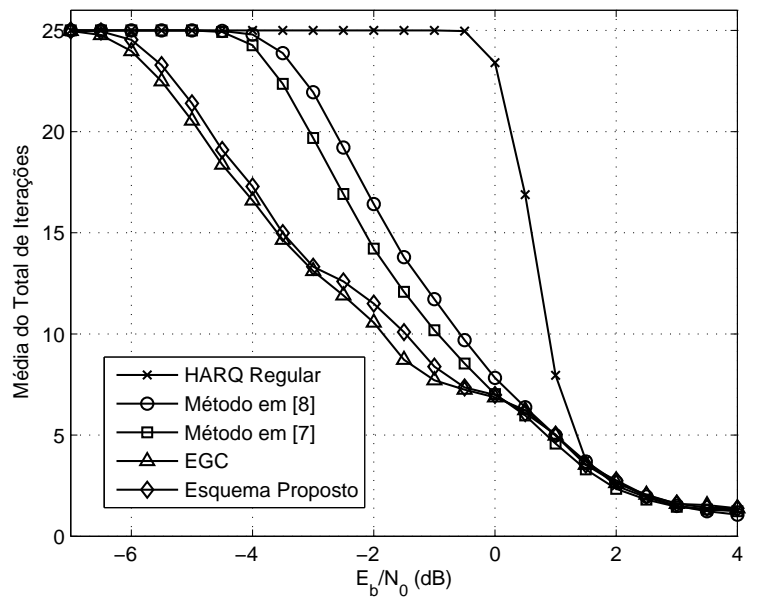

Fig. 3. Número médio do total de iterações versus $E_{b} / N_{0}$ para o esquema proposto, HARQ com EGC, o método em [7], o esquema em [8], e para HARQ regular sem combinação. A taxa do código é $R=1 / 2$.

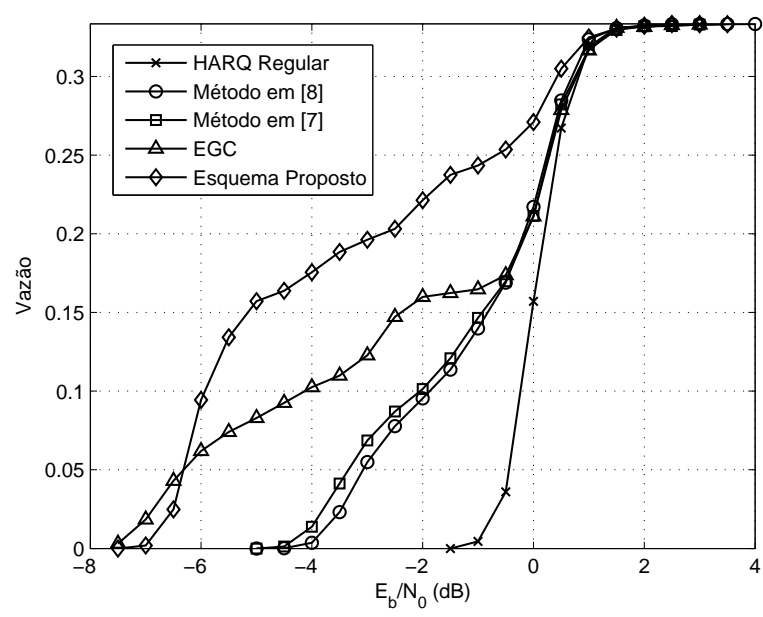

Fig. 4. Vazão versus $E_{b} / N_{0}$ para o esquema proposto, HARQ com EGC, os métodos em [7], [8] e HARQ regular sem combinação. A taxa do código é $R=1 / 3$.

na complexidade de decodificação.

Testes para outros tamanhos de bloco ( $L=97$ e $L=1024)$ demonstraram que o desempenho relativo entre os métodos são muito similares aos obtidos para o comprimento do bloco de informação $L=256$ bits. Além disto, outras técnicas diferentes de transmissão das sugeridas na Seção III foram também testadas, mas não se mostraram superiores à considerada neste artigo.

Uma desvantagem do método introduzido aqui, para qualquer taxa, é que o amplificador de potência do transmissor deve ser capaz de aumentar sua potência de saída instantânea sem distorcer o sinal. Assim, o back-off de saída do amplificador tem que ser maior do que o usual. Caso ocorra algum tipo de distorção, os ganhos sobre os outros métodos serão menores. O esquema proposto seria então mais aplicável no downlink, onde apenas uma estação base atende vários

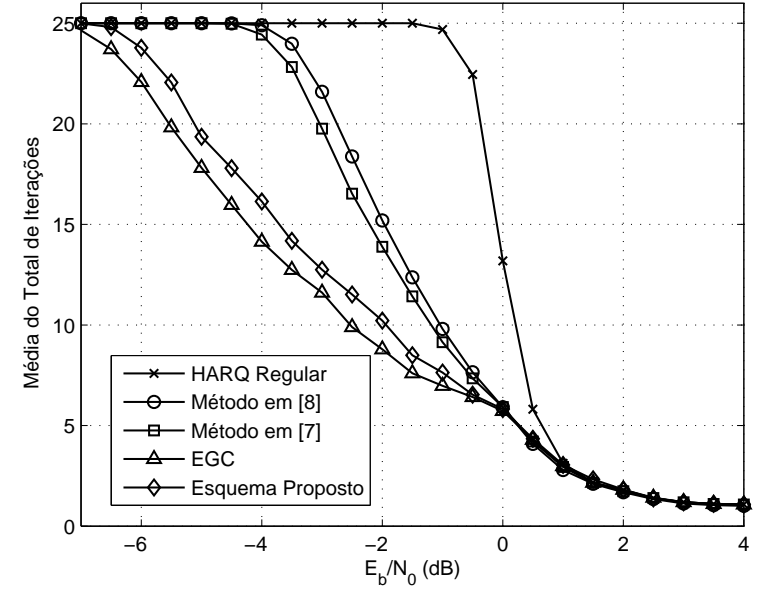

Fig. 5. Número médio do total de iterações versus $E_{b} / N_{0}$ para o o esquema proposto, HARQ com EGC, o método em [7], o esquema em [8], e para HARQ regular sem combinação. A taxa do código é $R=1 / 3$.

usuários. Assim, a restrição mais rígida no projeto do amplificador de potência é atribuída apenas à estação base.

\section{AnÁlise BASEAdA EM Evolução DA DEnsidAde}

Nesta seção conduziremos uma análise baseada em evolução da densidade [19], que denominaremos por "DE", para investigar as razões por que o esquema proposto supera o HARQ EGC clássico. Nós apresentamos as análises para o caso de taxa $R=1 / 2$ por questões de brevidade, e porque os resultados são muito similares para o caso de taxa $R=1 / 3$. Utilizamos a aproximação Gaussiana do modelo DE definido em [19]. Nas seguintes análises, $\operatorname{SNR}(i)_{\text {in }}$ e $\operatorname{SNR}(i)_{\text {out }}$ significam a relação sinal ruído para a qualidade da informação extrínseca na entrada e na saída do $i$-ésimo componente do decodificador, $i=1,2$. As SNRs são calculadas como $\mu / 2$, onde $\mu$ é a média da densidade Gaussiana simétrica [19], que aproxima-se do comportamento das mensagens de informação extrínseca na entrada e saída dos componentes do decodificador em um decodificador turbo. Além disso, seguindo a notação de [19], definimos duas funções não lineares $G 1$ e $G 2$, tal que $\mathrm{SNR} 1_{\text {out }}=G 1\left(\mathrm{SNR} 1_{\text {in }}, E_{b} / N_{0}\right)$ e $\mathrm{SNR} 2_{\text {out }}=$ $G 2\left(\mathrm{SNR} 2_{i n}, E_{b} / N_{0}\right)$. Além disto, $\mathrm{SNR} 2_{\text {in }}=\mathrm{SNR} 1_{\text {out }}$.

A Figura 6 apresenta a análise DE para o caso do esquema proposto e de HARQ com EGC, para $E_{b} / N_{0}=0 \mathrm{~dB}$ e duas transmissões (transmissão original mais uma retransmissão). $\mathrm{Na}$ figura nós podemos ver que os dois esquemas convergem, uma vez que os dois túneis de decodificação iterativa estão bem abertos. O túnel para o HARQ com EGC está mais aberto do que o do esquema proposto porque no caso do HARQ com EGC mais informação foi enviada na segunda transmissão. No caso do esquema proposto apenas bits sistemáticos com energia amplificada foram enviados. É importante dizer que o túnel está fechado para os dois métodos depois da primeira transmissão, de forma que a segunda transmissão sempre será necessária para este valor de $E_{b} / N_{0}$. Como consequência, a vazão $T$ para o HARQ com EGC será $T=\frac{L}{2 L+2 L}=\frac{1}{4}$, levando em conta que dois pacotes de $2 L$ bits codificados 
são enviados para entregar $L$ bits de informação. Para o esquema proposto a vazão é $T=\frac{L}{2 L+L}=\frac{1}{3}$, sendo que um pacote de $2 L$ bits codificados e um pacote adicional de $L$ bits são enviados para entregar $L$ bits de informação. Tais valores de vazão são exatamente os mesmos daqueles obtidos na Figura 2 para este valor de $E_{b} / N_{0}$. Assim, a vantagem do esquema proposto vem do fato de que, mesmo enviando menos bits codificados, ele também é capaz de abrir o túnel de decodificação iterativa e alcançar a convergência.

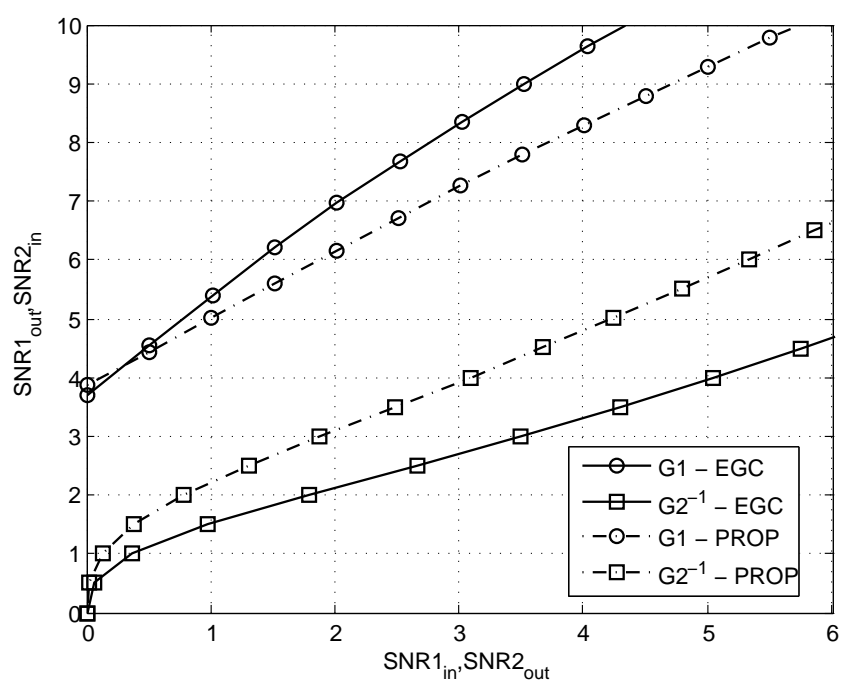

Fig. 6. Análise DE para o esquema proposto (PROP) e HARQ com EGC (EGC). A taxa do código é $R=1 / 2, E_{b} / N_{0}=0 \mathrm{~dB}$, e duas transmissões foram realizadas.

A Figura 7 apresenta a mesma análise, mas para o caso de $E_{b} / N_{0}=-2.75 \mathrm{~dB}$ e três transmissões (transmissão original mais duas retransmissões). Os dois túneis de decodificação estão fechados para menos transmissões, tal que três transmissões são sempre necessárias para este valor de $E_{b} / N_{0}$. Assim, a vazão para o HARQ com EGC é $T=\frac{L}{2 L+2 L+2 L}=$ $\frac{1}{6}$, enquanto que a vazão para o esquema proposto é $T=$ $\frac{L}{2 L+L+L}=\frac{1}{4}$. Tais valores de vazão estão novamente de acordo com aqueles apresentados na Figura 2. Além disto, o fato do túnel de decodificação iterativa ser sempre mais largo nas Figuras 6 e 7 ajuda a explicar por que o HARQ com EGC em geral requer um pouco menos iterações do que o esquema proposto, como apresentado na Figura 3.

A Figura 8 considera o caso do $E_{b} / N_{0}=-5.0 \mathrm{~dB}$ e quatro transmissões (transmissão original mais três retransmissões). Da figura nós podemos ver que o HARQ com EGC já converge com apenas quatro transmissões, enquanto que para o esquema proposto o túnel de decodificação iterativa ainda está fechado. Isto acontece porque a quantidade reduzida de informação que retransmitimos no esquema proposto não foi suficiente para abrir o túnel para este valor de $E_{b} / N_{0}$. A Figura 9 apresenta o caso de $E_{b} / N_{0}=-5.0$ e cinco transmissões, para o esquema proposto, e o esquema proposto mas sem controle de potência (sem alocação adicional de energia nas retransmissões). $\mathrm{Na}$ figura podemos ver que o esquema proposto converge depois da quinta transmissão. Podemos também observar quão importante é a operação realizada pelo mecanismo de controle

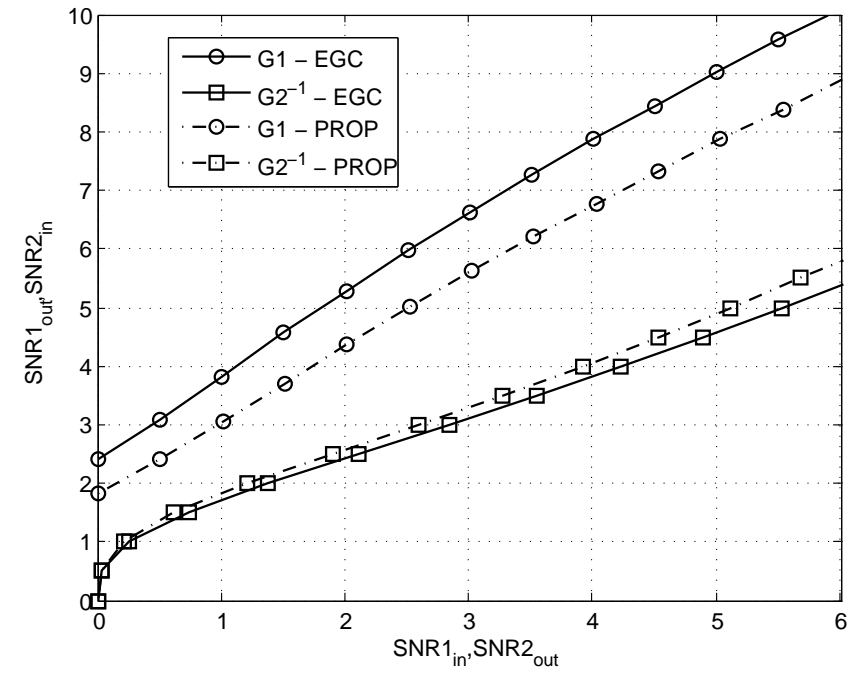

Fig. 7. Análise DE para o esquema proposto (PROP) e HARQ com EGC. A taxa do código é $R=1 / 2, E_{b} / N_{0}=-2.75 \mathrm{~dB}$, e três transmissões foram realizadas.

de potência em nossa proposta. Sem controle de potência não seria possível a convergência mesmo na quinta transmissão. Nas Figuras 8 e 9 podemos estimar a vazão do HARQ com EGC como $T=\frac{L}{2 L+2 L+2 L+2 L}=\frac{1}{8}$ e do esquema proposto como $T=\frac{L}{2 L+L+L+L+L}=\frac{1}{6}$, que uma vez mais corrobora os resultados da Figura 2. Note que mesmo necessitando mais transmissões, o esquema proposto envia menos bits adicionais para abrir o túnel de decodificação iterativa, e com isto alcança uma vazão maior.

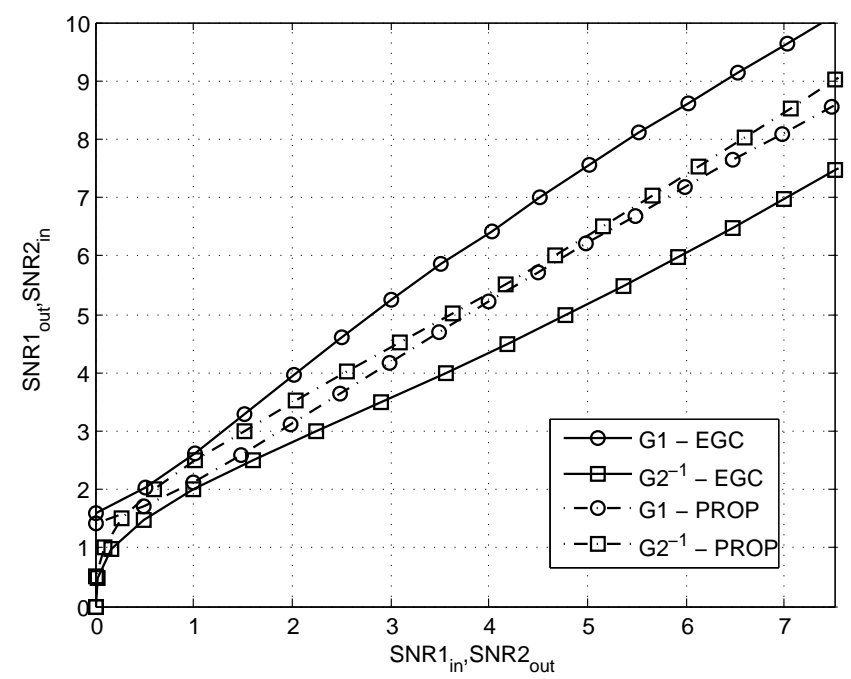

Fig. 8. Análise DE para o esquema proposto (PROP) e HARQ com EGC (EGC). A taxa de código é $R=1 / 2, E_{b} / N_{0}=-5.0 \mathrm{~dB}$, e quatro transmissões foram realizadas.

Das análises acima, podemos dizer que o esquema proposto supera o HARQ com EGC clássico porque ele pode alcançar a convergência com menos bits transmitidos. Além disto, é importante ressaltar que, mesmo aumentando a energia durante as retransmissões, a energia total média gasta em todo o 


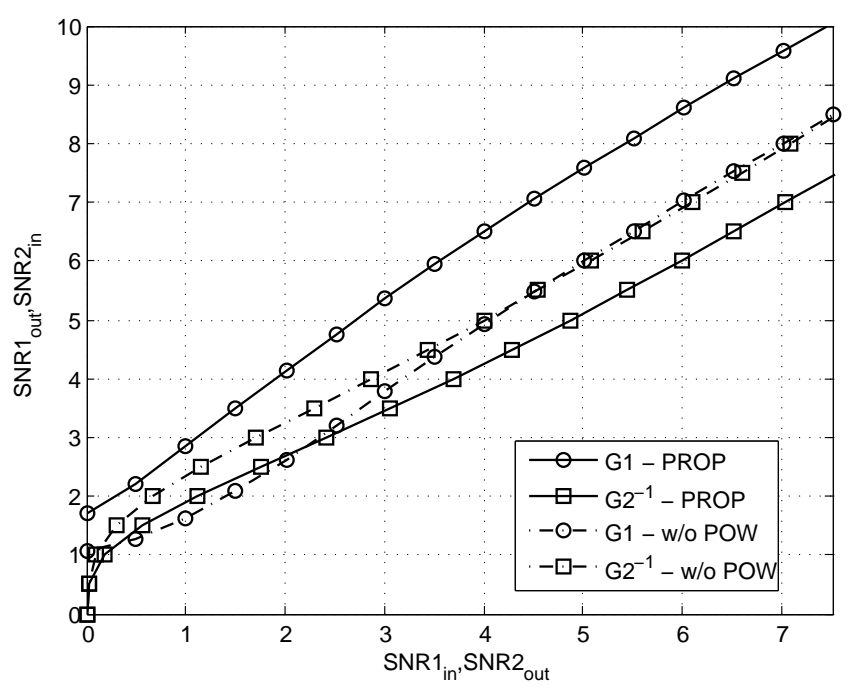

Fig. 9. Análise DE para o esquema proposto (PROP) e para o esquema proposto sem controle de potência (w/o POW). A taxa do código é $R=1 / 2$, $E_{b} / N_{0}=-5.0 \mathrm{~dB}$, e cinco transmissões foram realizadas.

processo pelo método proposto é a mesma que no caso do HARQ com EGC. Desta forma, os ganhos não vêm devido a um uso extra de energia, mas pela alocação apropriada dos recursos existentes.

\section{COMEnTÁrios FinAis}

Apresentamos um novo esquema de HARQ usando códigos turbo, controle de potência e combinação por diversidade. Os resultados das simulações computacionais demonstraram que o novo método supera o esquema clássico de HARQ usando EGC e também os esquemas de combinação de informação suave. Além do mais, conduzimos uma análise baseada em evolução da densidade para justificar as razões pelas quais o método proposto supera o HARQ com EGC mesmo retransmitindo menos informação adicional. Ficou claro que o melhor desempenho do método proposto vem da melhor alocação dos recursos disponíveis, quando comparado com o HARQ com EGC clássico. Um ponto importante a ser explorado em trabalhos futuros é como um esquema baseado em transmissões parciais e controle de potência, como o proposto neste artigo, desempenha no caso de um canal Rayleigh ao invés do AWGN.

\section{AgRADECIMENTOS}

Este trabalho foi financiado em parte pelo CNPq, através dos processos 562164/2008-1, 143460/2008-0 e 303181/2007-9.

\section{REFERÊNCIAS}

[1] S. B. Wicker, Error Control Systems for Digital Communication and Storage, Englewood Cliffs: Prentice Hall, 1995.

[2] C. Berrou and A. Glavieux, "Near optimum error correcting coding and decoding: Turbo-codes," IEEE Trans. Commun., vol. 44, no. 10, pp. 1261-1271, Oct. 1996.

[3] 3GPP TS 25.308, ”3rd Generation Partnership Project; Technical Specification Group Radio Access Network; High Speed Downlink Packet Access (HSDPA); Overall description; Stage 2 (Release 5),’Mar. 2002.
[4] IEEE Standard 802.16e-2005, "IEEE Standard for local and metropolitan area networks Part 16: Air Interface for Fixed and Mobile Broadband Wireless Access Systems,'Feb. 2006.

[5] 3GPP TR 25.814, "3rd Generation Partnership Project; Technical Specification Group Radio Access Network; Physical layer aspects for evolved Universal Terrestrial Radio Access (UTRA) (Release 7),'June 2006.

[6] D. N. Rowitch and L. B. Milstein, "On the performance of hybrid FEC/ARQ systems using rate compatible punctured turbo (RCPT) codes," IEEE Trans. Commun., vol. 48, no. 6, pp. 948-959, Jun 2000.

[7] K. R. Narayanan and G. L. Stüber, "A novel ARQ technique using the turbo coding principle," IEEE Commun. Letters , vol. 1, no. 2, pp. 49-51, Mar. 1997.

[8] I. D. Holland, H.-J. Zepernick, and M. Caldera, "Soft combining for hybrid ARQ," IEE Electron. Lett., vol. 41, no. 22, pp. 1230-1231, Oct 2005.

[9] R. D. Souza, M. E. Pellenz, and T. Rodrigues, "Hybrid ARQ scheme based on recursive convolutional codes and turbo decoding," IEEE Trans. Commun., vol. 57, no. 2, pp. 315-318, Feb. 2009.

[10] D. Chase, "Code combining - A maximum-likelihood decoding approach for combining an arbitrary number of noisy packets," IEEE Trans. Commun., vol. 33, no. 5, pp. 385-393, May 1985.

[11] J. Hagenauer, "Rate-compatible punctured convolutional codes (RCPC codes) and their applications," IEEE Trans. Commun., vol. 36, no. 4, pp. 389-400, April 1988.

[12] Motorola, "Performance comparison of hybrid-ARQ schemes,"3GPP input paper TSGR1\#17(00)1396, Oct. 2000.

[13] P. Frenger, S. Parkvall, and E. Dahlman, "Performance comparison of HARQ with Chase combining and incremental redundancy for HSDPA," Proc. of the IEEE VTC Fall, vol. 3, pp. 1829-1833, Oct. 2001.

[14] K. C. Beh, A. Doufexi, and S. Armour, "Performance evaluation of hybrid ARQ schemes of 3GPP LTE OFDMA system," Proc. of the IEEE PIMRC, pp. 1-5, Sept. 2007.

[15] J.-F. Cheng, "Coding performance of hybrid ARQ schemes," IEEE Trans. Commun., vol. 54, no. 6, pp. 1017-1029, June 2006.

[16] D. Toumpakaris, J. Lee, A. Matache, H.-L. Lou, "Performance of MIMO HARQ under Receiver Complexity Constraints," Proc. of the IEEE Globecom, pp. 1-5, Dec. 2008.

[17] J. Lee, H.-L. Lou, D. Toumpakaris, E. Jang, J. Cioffi, 'Transceiver design for MIMO wireless systems incorporating hybrid ARQ," IEEE Commun. Magazine, vol. 47, no. 1, pp. 32-40, Jan. 2009.

[18] R. J. McEliece and W. Lin, "The trellis complexity of convolutional codes," IEEE Trans. Inform. Theory, vol. 42, no. 6, pp. 1855-1864, Nov. 1996.

[19] D. Divsalar, S. Dolinar, and F. Pollara, "Iterative turbo decoder analysis based on density evolution," IEEE Journal on Selected Areas in Communications, vol. 19, no. 5, pp. 891-907, May 2001.

[20] S. Kallel, "Complementary punctured convolutional (CPC) codes and their application," IEEE Trans. Commun., vol. 43, no. 6, pp. 20052009, June 1995. 